\title{
Fungal community in olive fruits of cultivars with different susceptibilities to anthracnose and selection of isolates to be used as biocontrol agents
}

\author{
Gilda Preto, Fátima Martins, José Alberto Pereira, Paula Baptista* \\ CIMO, School of Agriculture, Polytechnic Institute of Bragança, Campus de Santa Apolónia, 5300-253 Bragança, Portugal
}

\section{A R T I C L E I N F O}

\section{Keywords:}

Olea europaea

Colletotrichum acutatum

Endophytes

Epiphytes

Cultivar effect

Biocontrol

\begin{abstract}
A B S T R A C T
Olive anthracnose is an important fruit disease in olive crop worldwide. Because of the importance of microbial phyllosphere to plant health, this work evaluated the effect of cultivar on endophytic and epiphytic fungal communities by studying their diversity in olives of two cultivars with different susceptibilities to anthracnose. The biocontrol potency of native isolates against Colletotrichum acutatum, the main causal agent of this disease, was further evaluated using the dual-culture method. Fungal community of both cultivars encompassed a complex species consortium including phytopathogens and antagonists. Host genotype was important in shaping endophytic but not epiphytic fungal communities, although some host-specific fungal genera were found within epiphytic community. Epiphytic and endophytic fungal communities also differed in size and in composition in olives of both cultivars, probably due to differences in physical and chemical nature of the two habitats. Fungal tested were able to inhibited C. acutatum growth (inhibition coefficients up to 30.9), sporulation (from 46 to $86 \%$ ) and germination (from 21 to $74 \%$ ), and to caused abnormalities in pathogenic hyphae. This finding could open opportunities to select specific beneficial microbiome by selecting particular cultivar and highlighted the potential use of these fungi in the biocontrol of olive anthracnose.
\end{abstract}

\section{Introduction}

Olive anthracnose, a disease of olive fruits mainly caused by diverse fungi clustering in the Colletotrichum acutatum species complex, is one of the most serious constrains to the olive crop production worldwide (Cacciola et al., 2012). In several Mediterranean countries, such as Italy, Spain and Portugal, olive anthracnose was reported to cause 80-100\% yield loss due to premature fruit drop (Cacciola et al., 2012; Talhinhas et al., 2011). Anthracnose is also responsible for quality degradation of olive oils by increasing the free acidity and the peroxide values, and by decreasing oxidative stability and the contents on phenolic compounds (Moral et al., 2014). The use of copper fungicides has been the main control strategy adopted against this disease (Cacciola et al., 2012). Besides not being completely effective on preventing olive anthracnose (Moral et al., 2014), there are numerous environmental risks and toxicity problems associated with it because of possible copper residues in olive fruits and consequently in oil (Soares et al., 2006). Chemical control measures are also not compatible with sustainable production systems (organic and integrated production) which are the pillars of the European Model for Agriculture.

Fruit-associated microorganisms may be explored, in an integrative perspective, for designing novel and environmental-friendly control strategies for management of olive anthracnose. The above-ground parts of plants, and called phyllosphere, are colonized by a diverse microbial community that can inhabit the interior of plant tissues (as endophytes) as well as plant's surface (as epiphytes) (Vorholt, 2012). Recent studies indicate that host plant genotype can influence the microbiome composition by acting on keystone species, which in turn affects plant colonization by many other microbes (Agler et al., 2016). In this changed microbial community, species with diverse functional types, form complex, interconnected microbial networks that could have important implications for plant health (Heijden and Hartmann, 2016). These findings give rise to novel ideas for sustainable management of plant diseases. For instances, genotype selection offers opportunities to select for certain keystone taxa, which in turn might recruit beneficial organisms or prevent invasion of pathogens (Heijden and Hartmann, 2016). However, knowledge about the diversity of those plant-associated microbes as well as of how phyllosphere microorganisms interact among themselves and with their plant hosts is still very incomplete, which could jeopardize that strategy. This is especially true for the olive tree, being only recently analyzed the fungal community associated to their fruits (Abdelfattah et al., 2015). These researchers identified a complex fungal consortium, comprising pathogenic (e.g. Colletotrichum spp., Pseudocercospora spp.) and potentially antagonistic,

\footnotetext{
* Corresponding author.

E-mail address: pbaptista@ipb.pt (P. Baptista).
} 
that can have a significant impact on olive production. However, this study did not compare epiphytic and endophytic fungal communities on single olives. Epiphytes and endophytes are exposed to different stressors, which might have important effects on both fungal communities composition (Rastogi et al., 2013). Epiphytes were more challenged by environmental stressors, such as temperature, humidity and solar radiation, whereas plant defense reactions were the major challenge for endophytes (Rastogi et al., 2013).

In this work, we studied, for the first time, the culturable fungal epiphytes and endophytes inhabiting olives from two Portuguese cultivars with different susceptibilities to olive anthracnose: Madural cultivar is susceptible and Verdeal Transmontana is moderately tolerant to olive anthracnose. We wanted to determine whether some fungi can systemically colonize olives of the two cultivars or whether such cultivar eventually develops their own specific fungal communities. The inhibitory efficacy of several fungi isolated from both cultivars against the phytopathogenic $C$. acutatum was additionally evaluated, under in vitro conditions. We wanted to determine whether inhibitory efficacy displayed by fungi is dependent of their origin (i.e. olive cultivar) and to find new fungi with potential to be explored as biocontrol agents against $C$. acutatum.

\section{Material and methods}

\subsection{Sample collection}

Olive fruits were collected in three organic olive (Olea europaea L.) groves located in Mirandela (Northeast of Portugal, $41^{\circ} 33^{\prime} 29.6^{\prime \prime} \mathrm{N} 7^{\circ} 08^{\prime} 45.9^{\prime \prime} \mathrm{W}$ ), in November of 2015 . Each one comprises two different Portuguese olive cultivars, Verdeal-Transmontana and Madural, with trees of medium size (ages ranging 60 years) and planted at $7 \times 7 \mathrm{~m}$ spacing. In each grove, seven olive trees of each cultivar were randomly selected. From each tree, five symptomless olive fruits, at a maturity index ranging from 1 to 3 (Hermoso et al., 2001), were aseptically handpicked all around the perimeter of the tree at the operator height, and placed directly into sterile bags. The olives were transported to the laboratory in an icebox, and stored at $4{ }^{\circ} \mathrm{C}$ until isolation of epi- and endophytic fungi the following day.

\subsection{Isolation of epiphytic and endophytic fungi from olives}

Epiphytic fungi were obtained by imprinting the intact olive fruits samples gently on Potato Dextrose Agar (PDA, Difco) plates supplemented with $0.01 \%(\mathrm{w} / \mathrm{v})$ chloramphenicol (Oxoid, Basingstoke, Hampshire, UK). Olives were rolled over the agar surface (one fruit per plate), being used a total of 210 fruits (5 olives $\times 7$ olive trees $\times 3$ orchard $\times 2$ different cultivars) for the isolation of epiphytes. Endophytic fungi were obtained from the same olives used to isolated epiphytes, after surface sterilization of fruit tissues. Surface sterilization was performed through sequential immersion of fruits in $70 \%(\mathrm{v} / \mathrm{v})$ ethanol ( $1 \mathrm{~min}), 3 \%(\mathrm{v} / \mathrm{v})$ sodium hypochlorite $(2 \mathrm{~min}), 70 \%(\mathrm{v} / \mathrm{v})$ ethanol $(1 \mathrm{~min})$, and further rinsed three times with sterile distilled water (one min each). After being dried on sterile filter paper, each fruit was cut into five segments (ca. $5 \times 5 \mathrm{~mm}$ ), and transferred into the same medium used to isolated epiphytes (PDA). Therefore a total of 1050 segments ( 5 olives $\times 5$ segments per olive $\times 7$ olive trees $\times 3$ orchard $\times 2$ different cultivars) were inoculated. Validation of the surface sterilization procedure was done by imprinting the surface sterilized fruit tissues onto PDA media. Plates were incubated at $25 \pm 2{ }^{\circ} \mathrm{C}$ in the dark and were daily observed for microbial growth and colonies counting, over a period of up to two months. Single colonies were picked and cultivated on new PDA plates to obtain pure isolates for subsequent identification. Results of epiphytes were expressed as $\mathrm{CFU} / \mathrm{cm}^{2}$, i.e. the number of individual colonies of fungi adhered to fruit surface. For the approximate calculus of fruit surface area was used the prolate spheroid formula (Weisstein, 2013) from the longitudinal and transverse axes of fruits. For the Verdeal Transmontana and Madural fruits used in the present study, the average area was $11.61 \mathrm{~cm}^{2}$ and $9.88 \mathrm{~cm}^{2}$, respectively. Pure cultures of each isolate were preserved and deposited in the culture collection of the Polytechnic Institute of Bragança (School of Agriculture).

\subsection{Identification of fungal isolates}

Fungal isolates were identified by using morphological and molecular approaches. At first, groups of strains were formed according their morphological similarity (culture colony, hyphae, spores and reproductive structures), and then one representative isolate of each morphotype was selected for molecular identification, using internal transcribed spacer (ITS) region of the nuclear ribosomal DNA (rDNA). Total genomic DNA was extracted from mycelial/spores using the REDExtract-N-Amp ${ }^{\mathrm{TM}}$ Plant PCR kit (Sigma, Poole, UK). The ITS region (ITS1, 5.8S, ITS2) was amplified using the universal ITS1 and ITS4 primers in a PCR protocol formerly described by Oliveira et al. (2012). The amplified products ( $\sim 650 \mathrm{bp}$ ) were purified and sequenced using Macrogen Inc. (Seoul, South Korea) services, using the same primers to initiate the reaction. The obtained DNA sequences were analyzed with DNASTAR v.2.58 software, and fungal identification was performed using both NCBI (http://www.ncbi.nlm.nih.gov) and UNITE (https:// unite.ut.ee/) databases and BLAST algorithm. Blast results were sorted according to the higher identity score and the lowest E-value. For sequence identities $>98 \%$, the genus and species were accepted; for sequence identities between $95 \%$ and $97 \%$, only the genus was accepted; and for sequence identities $<95 \%$, isolates were labelled as 'unknown' fungi. The sequences obtained are available at GenBank (accession numbers KY001582-KY001603; KY001609-KY001612). Each operational taxonomic unit (OTU) was taxonomically classified according to the Index Fungorum Database (www.indexfungorum.org).

\subsection{Diversity and composition of fungal communities}

Occurrence of fungal endophytes and epiphytes was measured by calculating the frequency of colonization (FC, \%) and relative abundance (RA, \%), respectively. The frequency of colonization was calculated as the total number of olive tissue segments colonized by each endophyte divided by the total number of olive segments surveyed. The relative abundance was determined as the total number of isolates of an OUT/genus divided by the total number of isolates.

The diversity of fungal endophytes and epiphytes was evaluated at the level of their richness (total number of different OTUs, and average number of OTUs per tree), their abundance (total number of isolates, and average number of isolates per tree), and also by computing Shannon-Wiener $(\mathrm{H})$ index species diversity, which accounts for both abundance and evenness of the species present (Magurran, 2004). This index was computed in Species Diversity and Richness v. 4.0 software (Seaby and Henderson, 2006). Species-richness estimation was also performed by using species accumulation curves, which were calculated using the sample-based rarefaction index (Mao Tau) and computed in EstimateS v. 9.1.0 (Colwell, 2013) using 1000 runs of bootstrapping with replacement. Results of diversity and abundance were presented as the mean of 21 independent experiments displaying the respective SE values or as the total number (the values for all samples lumped together). Differences among the means were determined by an analysis of variance (ANOVA) with SPSS v.21 software, and the averages were compared using Tukey's test $(\mathrm{p}<0.05)$.

Non-metric multidimensional scaling (NMDS) was carried out to explore the similarity of fungal's community among olive tree cultivars (Madural and Verdeal Transmontana). NMDS was performed by using Jaccard's and Bray-Curtis similarities matrices, because they consider different kinds of information. Jaccard's index only compares presence or absence of taxa among samples, disregarding species abundance (Magurran, 2004). Bray-Curtis index compares taxa presence or ab- 
sence and abundance among samples. This coefficient ignores cases in which the species is absent in both community samples, and is strongly influenced by the abundant species so rare species add very little influence (Bray and Curtis, 1957). The ordination analyses were conducted on presence/absence data (Jaccard's index) and raw data (Bray-Curtis coefficient). Analysis of similarity (ANOSIM) was used to test for significant differences $(p<0.05$ ) between fungal community groupings obtained in NMDS ordination, using the Bray-Curtis distance matrices. This analysis compares species composition between-groups (olive tree cultivars) and generates an R-value that gives the degree of discrimination between groups. When a significant difference was observed, similarity percentage analyses (SIMPER) were performed to reveal which OTUs contributed to the dissimilarity between olive tree cultivars. All multivariate analyses were done using the Community Analysis Package v. 5.0 (Henderson and Seaby, 2014).

\subsection{Antagonistic tests}

The screening of fungal strains for antagonistic activity against $C$. acutatum was only performed for those species: i) with exclusively occurrence to one of the cultivars; ii) that are easily grown, after several subcultures, in artificial media; iii) and that were not previously considered as pathogenic to plants. According to these criteria's, seven species were selected: Chondrostereum purpureum, Chaetomium globosum, Aspergillus westerdijkiae and Aspergillus sp. 1, all isolated from cv. Madural, and Quambalaria cyanescens, Epicoccum nigrum and Aspergillus brasiliensis isolated from cv. Verdeal Transmontana (please see results section). With the exception of $C$. purpureum all other fungi tested were isolated from the epiphytic flora. Biocontrol potential of these species against $C$. acutatum was assessed by using dual-culture method. Fungi were grown in PDA medium, for seven days at $25 \pm 2{ }^{\circ} \mathrm{C}$ in the dark, in order to provide mycelium/spores for the establishment of dual cultures. After that, mycelial discs ( $5 \mathrm{~mm}$ diameter) of each tested fungi and $C$. acutatum were removed aseptically from the colony margins and inoculated $3 \mathrm{~cm}$ apart on the surface of Petri dishes $(9 \mathrm{~cm}$ diameter) containing $10 \mathrm{ml}$ of PDA medium. Inoculation of fungi belonging to the genera Aspergillus and Quambalaria, was performed with $10 \mu \mathrm{L}$ of a spore suspension $\left(1 \times 10^{6}\right.$ spore $/ \mathrm{mL}$, in $0.025 \%$ aq. Tween 80$)$, due to their high capacity to sporulate. Controls consisted of PDA plates containing two inocula of the same taxa. Plates were incubated at $25 \pm 2{ }^{\circ} \mathrm{C}$ in the dark, and each treatment was replicated five times. During interaction, the radial growth towards (internal radius) the interacting fungus and the distance between plant pathogen and tested fungi colonies were measured daily by using a graduated ruler, until the pathogen/fungi had reached the edge of the plate. With the data obtained was calculated the maximum radial growth rate of the pathogen $\left(\mathrm{A}\right.$, in $\mathrm{cm}_{\text {day }}{ }^{-1}$ ). When the pathogen came into contact with the tested fungi, measurements of radial extension made after this time were used to determine pathogen growth rates in the zone-of-mixed culture (D). The inhibitory activity of each tested fungi was calculated according to the equation devised by Cray et al. (2015): Inhibition coefficient = $[(100-\mathrm{B}) \times 0.4]+[(100-\mathrm{C}) \times 0.4]+[(100-\mathrm{E}) \times 0.2]$, where $\mathrm{B}$ is the growth-rate $\mathrm{A}$ as a percentage of radial extension of pathogen in control cultures, $\mathrm{C}$ is the distance travelled by the pathogen as a percentage of the distance between the sites of inoculation, and $\mathrm{E}$ is the growth-rate $\mathrm{D}$ as a percentage of radial extension of pathogen in control cultures. According to this equation, $[(100-\mathrm{B}) \times 0.4]$ represents a potential $40 \%$ contribution of distal inhibition (prior to contact) of growth rate of the pathogen; $[(100-\mathrm{C}) \times 0.4]$ represents a potential $40 \%$ contribution of prevention of development of the pathogen colony in the vicinity of the tested fungi; and $[(100-E) \times 0.2]$ represents a potential $20 \%$ contribution of ability to inhibit pathogen growth rate when in a zone-of-mixed culture (Cray et al., 2015). Significant differences on inhibition coefficient among the fungi tested were verified using one-way ANOVA with a Tukey test at $p$-value $<0.05$, conducted with SPSS v.21 software.
At the end of the dual culture assays, both sporulation and spore viability of $C$. acutatum in the interaction zone with the tested fungi were evaluated. For the assessment of sporulation, a spore suspension were prepared by vortexing three $C$. acutatum mycelial plugs removed from the interaction zone, in $1 \mathrm{ml}$ of sterile $0.025 \%(\mathrm{v} / \mathrm{v}$ ) Tween-80. The concentration of spores in this suspension (spores $/ \mathrm{mL}$ ) was then determined with a Neubauer counting chamber. Germination, as a measure of viability, was assessed by inoculating 9-cm Petri dishes containing water agar ( $15 \mathrm{~g} / \mathrm{L}$ agar-agar) with the same spore suspension used to quantify sporulation. After incubation, at $25^{\circ} \mathrm{C} \pm 2{ }^{\circ} \mathrm{C}$ in the dark for $12-24 \mathrm{~h}$, the percentage of germination was evaluated microscopically by counting the number of germinated spores. The ability of the tested fungi to reduced $C$. acutatum sporulation and viability was evaluated by computing the percentage of inhibition in relation to control. Percentage inhibition was calculated as difference between control and treatment, divided by the control value and, multiplied by 100 .

\subsection{Macroscopic and microscopic analysis of the dual-culture interactions}

The interaction between fungi-phytopathogen was described according to the following categories: (1) contact inhibition, when growth of both species stops at the line of contact (no clear zone is formed); (2) inhibition at distance, when neither species can enter the area inhabited by the other (a clear zone is formed); (3) overgrowth of a mycelium over the other; (4) replacement, where mycelia of one fungus was replaced by its opponent; and (5) mutual replacement, where both fungi gained some of the territory of the other (Boddy, 2000).

Hyphae morphology in the interaction zone was evaluated by light microscopy. Hyphal of the fungal colonies were removed from the interaction zone (bearing growing colonies) and transferred aseptically to sterile slides. The samples were examined under a light microscope (Leica DM 2000). Cultures of C. acutatum in PDA without epi-and endophytic fungi inoculation served as control.

\section{Results}

\subsection{Description of the community}

The isolation of both endo- and epiphyte fungi from all olives allowed the identification of 27 OTUs, 19 of which were identified up to the species level (Table S1). The ITS sequences of the remaining OTUs were not specified to the species level (7 OTUs) or even to the genus level ( 1 OTU). The identified species belonged to 17 genera and 14 families, all from the Ascomycota (22 OTUs), Basidiomycota (3 OTUs) and Zygomycota (2 OTUs) phyla. Species accumulation curves obtained for total endophytes and epiphytes reach an asymptote, suggesting that the sampling effort was sufficient to discover most of the fungal species inhabiting olives of both cultivars (Fig. 1).

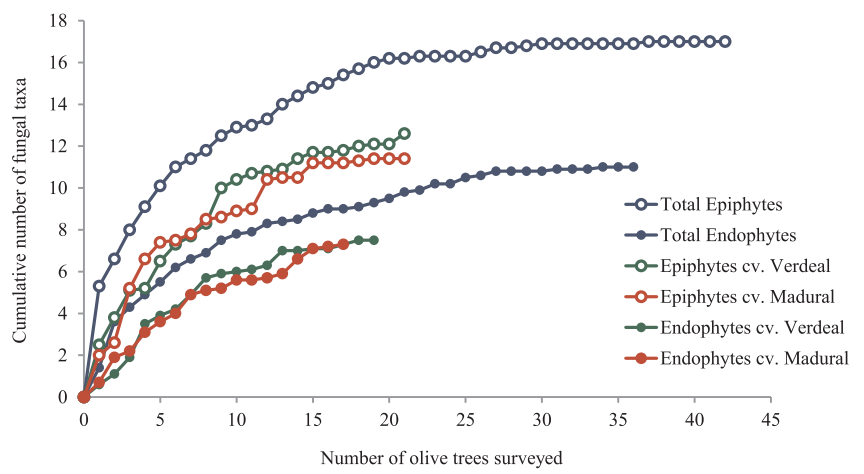

Fig. 1. Species accumulation curves for all fungal endophytes and epiphytes isolated from olives collected from 42 olive trees (Olea europaea L.) of cultivars Verdeal Transmontana and Madural. 
Table 1

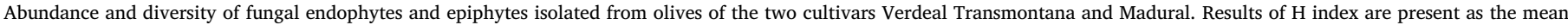
value \pm SD $(n=21)$ and as total number (in brackets). Different superscript lower case letters denote statistically significant differences ( $\mathrm{p}<0.05)$ between cultivars.

\begin{tabular}{|c|c|c|c|c|c|}
\hline & Parameters & & Verdeal Transmontana & Madural & Total \\
\hline \multirow[t]{6}{*}{ Endophytes } & Total no. of isolates & & 63 & 41 & 104 \\
\hline & Average no. of isolates per tree & & $3.00 \pm 0.51^{\mathrm{a}}$ & $1.95 \pm 0.38^{\mathrm{b}}$ & $2.48 \pm 0.35^{\mathrm{ab}}$ \\
\hline & Total no. of OTUs & & 7 & 8 & \\
\hline & Average no. of OTUs per tree & & $1.62 \pm 0.25^{\mathrm{a}}$ & $1.29 \pm 0.21^{\mathrm{a}}$ & $1.45 \pm 0.17^{\mathrm{a}}$ \\
\hline & Frequency of colonization (\%) & & 11.9 & 7.8 & 9.9 \\
\hline & Shannon-Wiener index $(\mathrm{H})$ & & $\begin{array}{l}0.6 \pm 0.13^{\mathrm{a}} \\
(1.6)\end{array}$ & $\begin{array}{l}0.4 \pm 0.11^{\mathrm{a}} \\
(1.7)\end{array}$ & $\begin{array}{l}0.5 \pm 0.12^{\mathrm{a}} \\
(1.8)\end{array}$ \\
\hline \multirow[t]{7}{*}{ Epiphytes } & Total no. of isolates & (cfu) & 503 & 493 & 996 \\
\hline & & $\left(\mathrm{cfu} / \mathrm{cm}^{2}\right)$ & 8.67 & 9.97 & 18.64 \\
\hline & Average no. of isolates per tree & (cfu) & $22.3 \pm 2.68^{\mathrm{a}}$ & $23.1 \pm 3.54^{\mathrm{a}}$ & $22.7 \pm 2.4^{\mathrm{a}}$ \\
\hline & & $\left(\mathrm{cfu} / \mathrm{cm}^{2}\right)$ & $0.41 \pm 0.06^{\mathrm{a}}$ & $0.45 \pm 0.07^{\mathrm{a}}$ & $0.44 \pm 0.05^{\mathrm{a}}$ \\
\hline & Total no. of OTUs & & 15 & 12 & 18 \\
\hline & Average no. of OTUs per tree & & $5.00 \pm 0.42^{\mathrm{a}}$ & $3.95 \pm 0.30^{\mathrm{b}}$ & $4.48 \pm 0.24^{\mathrm{ab}}$ \\
\hline & Shannon-Wiener index $(\mathrm{H})$ & & $\begin{array}{l}1.1 \pm 0.08^{\mathrm{a}} \\
(1.5)\end{array}$ & $\begin{array}{l}0.8 \pm 0.07^{\mathrm{b}} \\
(1.1)\end{array}$ & $\begin{array}{l}0.9 \pm 0.06^{\mathrm{ab}} \\
(1.4)\end{array}$ \\
\hline
\end{tabular}

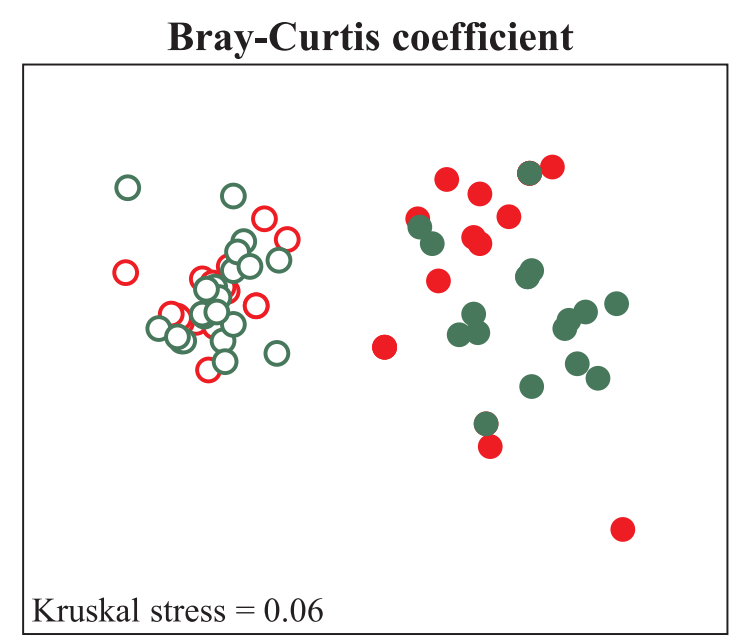

O Epiphytes cv. Madural

O Epiphytes cv. Verdeal Transmontana

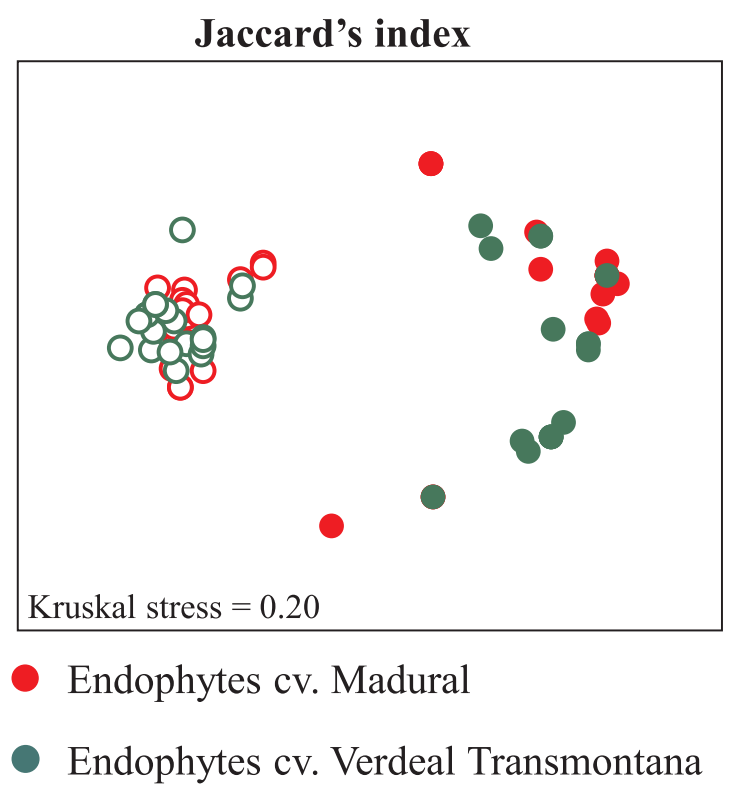

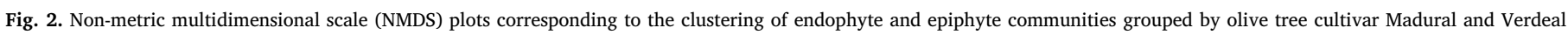

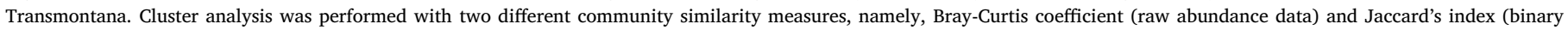
data). Kruskal's stress values less than 0.2 represent good ordination plots.

With regard to abundance, 1100 fungal isolates were obtained from all olives surveyed (Table 1), with the greatest percentage of the total isolates being from Ascomycota (95.5\%) and only 0.5\% being from Zygomycota. The most representative genera were Cladosporium (62.1\% of the total isolates), followed by Biscogniauxia $(8.1 \%)$ and Alternaria (6.9\%) (Fig. S1). Among the species identified in all analyzed olive tissues, Cladosporium cucumerinum and Biscogniauxia mediterranea were the most frequently isolated, collectively representing $68.0 \%$ of the total isolates (data not shown). Many species were represented by few isolates $(48.1 \%$ of the species were represented by only five or fewer isolates).

\subsection{Comparison of epiphytic and endophytic fungal community}

Overall, the diversity and abundance of fungal epiphytes was significantly $(\mathrm{p}<0.001)$ higher comparatively to endophytes (Table 1). In fact, the average number of epiphytic OTUs and isolates per tree was up to 3.1- and 9.1-fold significantly ( $p<0.001$ ) higher than of endophytes, respectively. Numbers of epiphytes of both cultivars were between 0.21 and $0.97 \mathrm{cfu} / \mathrm{cm}^{2}$ (avg. $0.44 \mathrm{cfu} / \mathrm{cm}^{2}$ ). Percentages of fruit colonization by endophytes were $9.9 \%$ (Table 1 ), when considering both cultivars.

Endophytic and epiphytic fungal communities are significantly different in terms of species compositions (ANOSIM $\mathrm{R}=0.850$, $\mathrm{p}=0.001$ ). The NMDS corroborated this result by showing a clear separation of the epiphytic and endophytic communities (Fig. 2). According to SIMPER analyses, both C. cucumerinum and B. mediterranea, contributing more than $58 \%$ of the average dissimilarity found among fungal communities. These two species were exclusively found in epiphytic samples and were by far the most abundant (Table S1). The high number of exclusive fungal OTUs associated to either endophytic (9) or epiphytic (17) fungal community could also account for the differences observed. Only one OTU (Cladosporium cladosporioides), out of the 27 recovered in this study, was common to both fungal communities (Table S1). Each of the fungal communities was also dominated by different OTUs. The most frequently isolated endophytes were members of the genera Gibberella and Neofabraea, collectively representing $75.0 \%$ of the total isolates, whereas within epiphytic community, Cladosporium, Biscogniauxia and Alternaria were the genera most frequently found, accounting for $83.5 \%$ of the total epiphytic isolates (Fig. 3). 


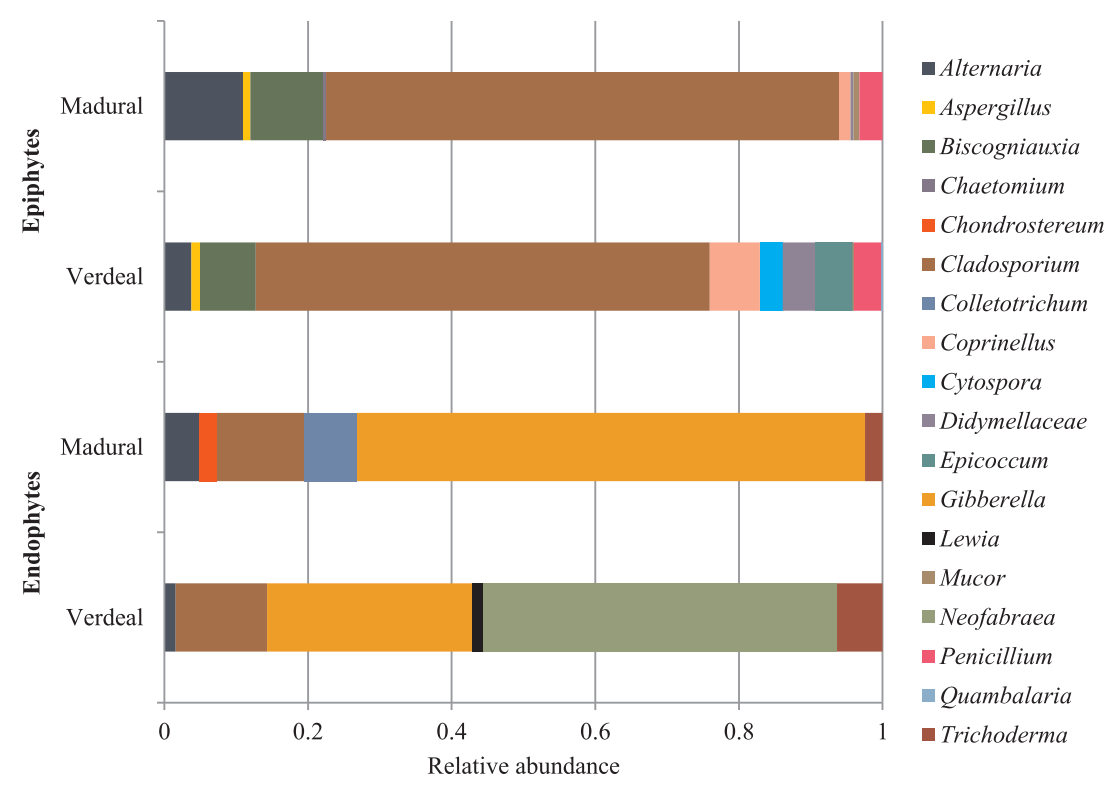

Fig. 3. Relative abundance of endophytic and epiphytic fungal genera/family associated with olives from cultivars Verdeal Transmontana and Madural.

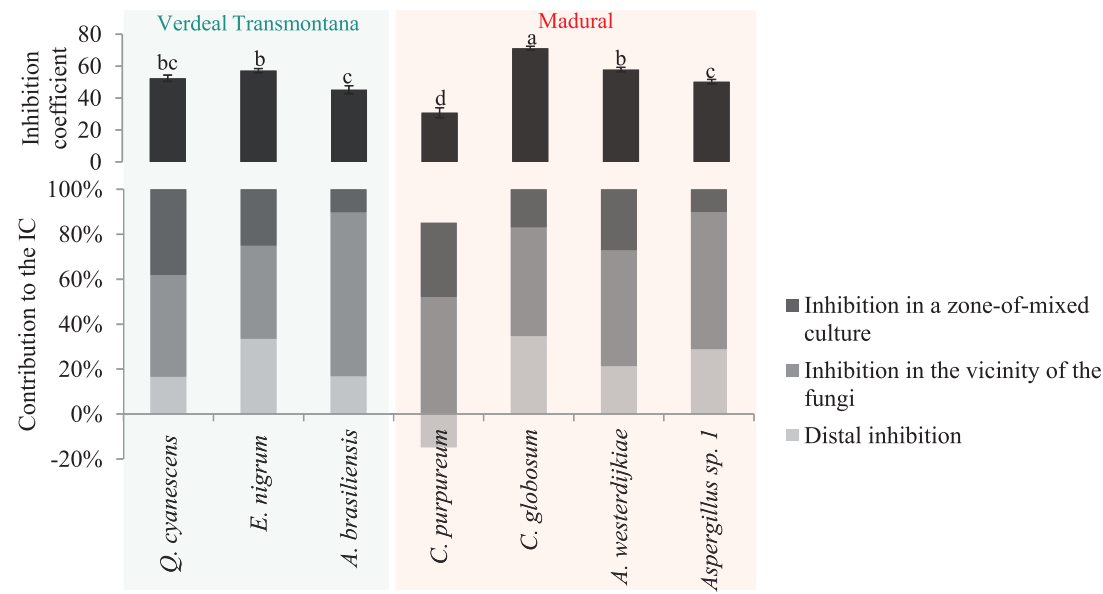

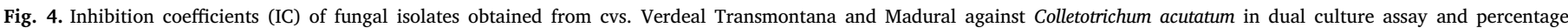

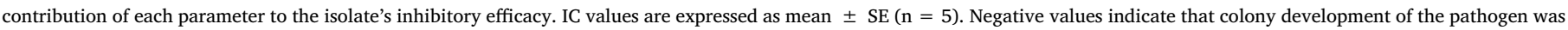
promoted. Bars with different lowercase letters indicate significant differences $(\mathrm{p}<0.05)$.

\subsection{Comparison of microbial communities between olive tree cultivars}

Species diversity differs significantly between olive tree cultivars only within epiphytic fungal community. Both the total number of epiphytic OTUs as well as the average number of epiphytic OTUs per tree was up to 1.3 -fold significantly $(\mathrm{p}<0.05$ ) higher on $\mathrm{cv}$. Verdeal Transmontana than on Madural (Table 1).

In what concerns abundance, only within endophytic community was found significant differences $(\mathrm{p}<0.05)$ among cultivars: a 1.5fold increase from Madural to Verdeal Transmontana (Table 1). The endophyte infection rates were similarly higher (up to 1.5-fold) in cv. Verdeal Transmontana than in Madural. Epiphytic fungal abundance did no differ significantly between cultivars, being ranging between 0.12 and $1.17 \mathrm{cfu} / \mathrm{cm}^{2}$, or between 0.24 and $1.50 \mathrm{cfu} / \mathrm{cm}^{2}$, in the $\mathrm{cv}$. Verdeal Transmontana and Madural, respectively.

The NMDS plots (Fig. 2) showed that only endophytic fungal communities composition differ significantly between cultivars (ANOSIM $R=0.467, p=0.001$ ), whereas epiphytic fungal communities composition of olives from cv. Madural was very similar to cv. Verdeal Transmontana (ANOSIM $\mathrm{R}=0.085, \mathrm{p}=0.014$ ). The differences in endophytic fungal communities were predominantly caused by the higher abundance of Gibberella genus (in particular of Gibberella baccata) in cv. Madural and the exclusive occurrence of Neofabraea vagabunda with high relative abundance in $\mathrm{cv}$. Verdeal Transmontana (Fig. 3). Although epiphytic community inhabiting olives of both cultivars overlapped, several fungal genera preferred either olives from cv. Madural (e.g. Chaetomium) or from cv. Verdeal Transmontana (e.g. Cytospora, Epicoccum and Quambalaria) (Fig. 3).

\subsection{Interaction between $C$ acutatum and fungal isolates}

All the fungal tested were able to inhibited $C$. acutatum growth, with inhibition coefficients ranging from 30.9 for $C$. purpureum to 71.3 for $C$. globosum, out of a theoretical maximum value of 100 (Fig. 4). Inhibition displayed by most of the fungi was promoted mostly in the vicinity of $C$. acutatum colonies, as evidenced by the high contribution of this parameter for the calculated inhibition coefficient values. This was particularly noticed for A. brasiliensis, Aspergillus sp. 1 and A. westerdijkiae. Inhibition at distance and after contact between colonies had lower contribution. Among the tested fungi, C. globosum was the most potent inhibitor at a distance, whereas $Q$. cyanescens was the most potent inhibitor following contact with pathogen.

Macroscopic examination of pairings showed that interactions can result in inhibition at mycelial contact, replacement and overgrowth of 

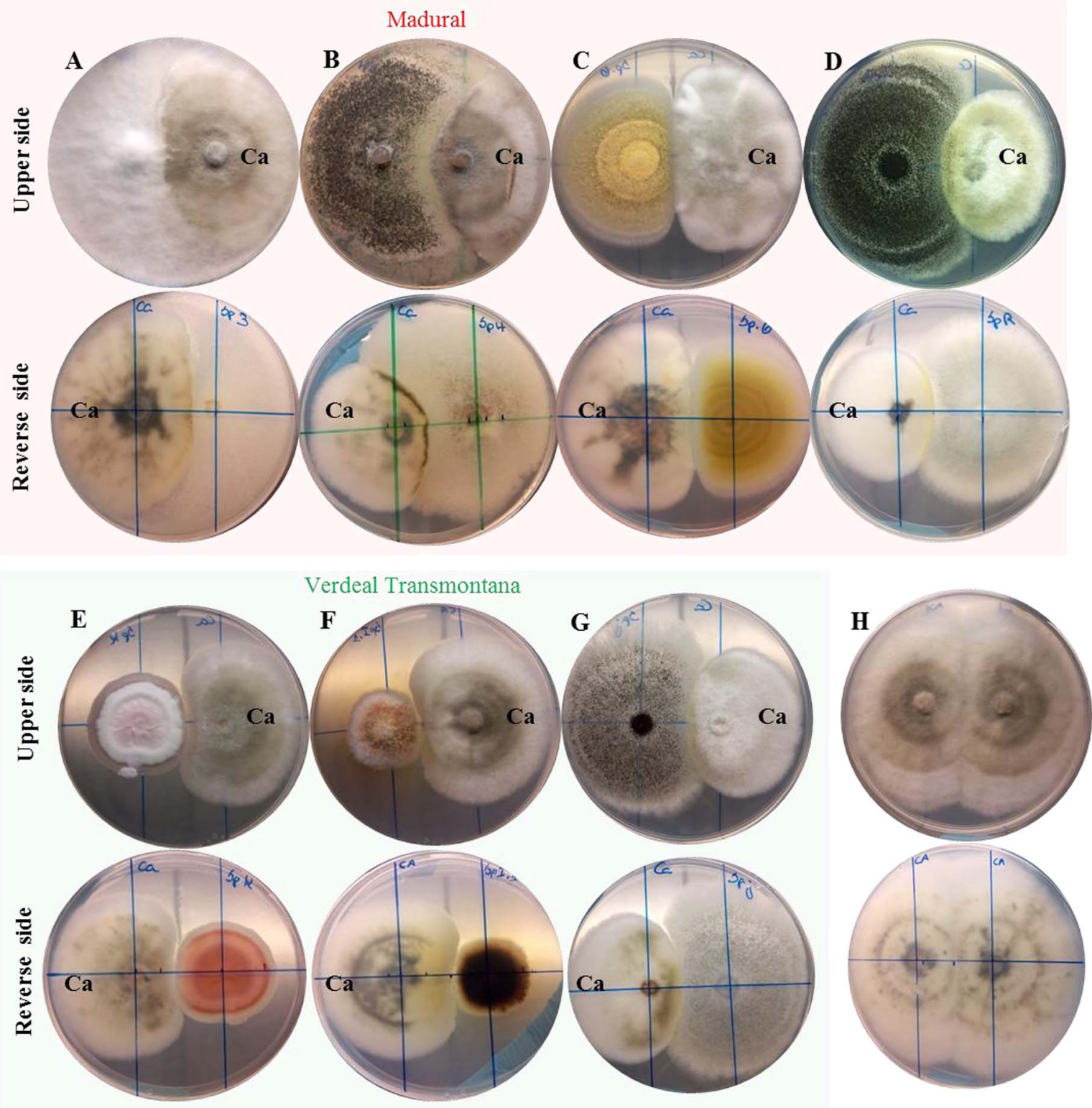

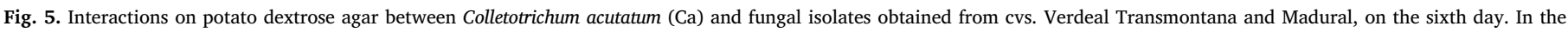

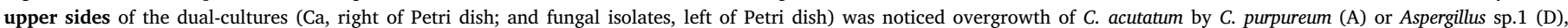

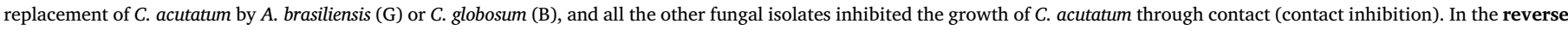

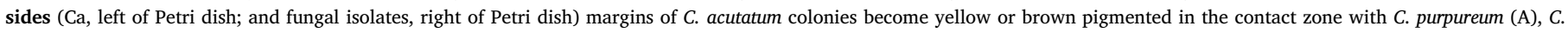
globosum (B), Aspergillus sp.1. (D), E. nigrum (F) and A. brasiliensis (G). H - Colletotrichum acutatum in control cultures.

C. acutatum by endo- or epiphytic fungi (Fig. 5). During interspecific mycelial interactions also occurred changes on mycelium pigmentation of $C$. acutatum, an effect that was not observed in the control. Margins of $C$. acutatum colonies become yellow or brown pigmented in the contact zone with E. nigrum, A. brasiliensis, C. purpureum, C. globosum and Aspergillus sp.1, which was best observed on the reverse side of the colony.

In all pairings was observed, in the interacting zones, alterations on C. acutatum hyphae morphology (Fig. 6). In contrast to control (Fig. 6E), hyphae of C. acutatum become swollen and distended in coculture with C. globosum (Fig. 6C) or collapsed and necrosed in dual cultures with A. brasiliensis, C. purpureum, Aspergillus sp.1 and $Q$. cyanescens (Fig. 6A and B). Collapse and twisting of $C$. acutatum hyphae were also the most common alterations observed in the vicinity of $A$. westerdijkiae and E. nigrum (Fig. 6D).

Effect of fungi isolates on $C$. acutatum sporulation and germination was also different depending on the species (Fig. 7). Three, out of the seven fungi tested (i.e. Q. cyanescens, E. nigrum and Aspergillus sp. 1) reduced significantly $(\mathrm{p}<0.05)$ the sporulation of $C$. acutatum by 46-86\% in comparison to control. In contrast, both C. globosum and A. westerdijkiae promoted significantly $(\mathrm{p}<0.05)$ the sporulation of $C$. acutatum (up to $135 \%$ when compared to control). All the tested fungi, with exception of $E$. nigrum and $C$. purpureum, reduced significantly ( $\mathrm{p}<0.05$ ) the conidial germination of $C$. acutatum by more than $21 \%$ in relation to the control, being the highest inhibition displayed by $Q$. cyanescens $(74 \%)$.

\section{Discussion}

Most studies on phyllosphere microorganisms have focused only on endophytic or epiphytic group, and have been restricted mostly to leaves (Lindow and Brandl, 2003). In the present work, the diversity of fungi inhabiting the surface and the inner tissues of olives from two different Portuguese cultivars (i.e. Verdeal Transmontana and Madural) 

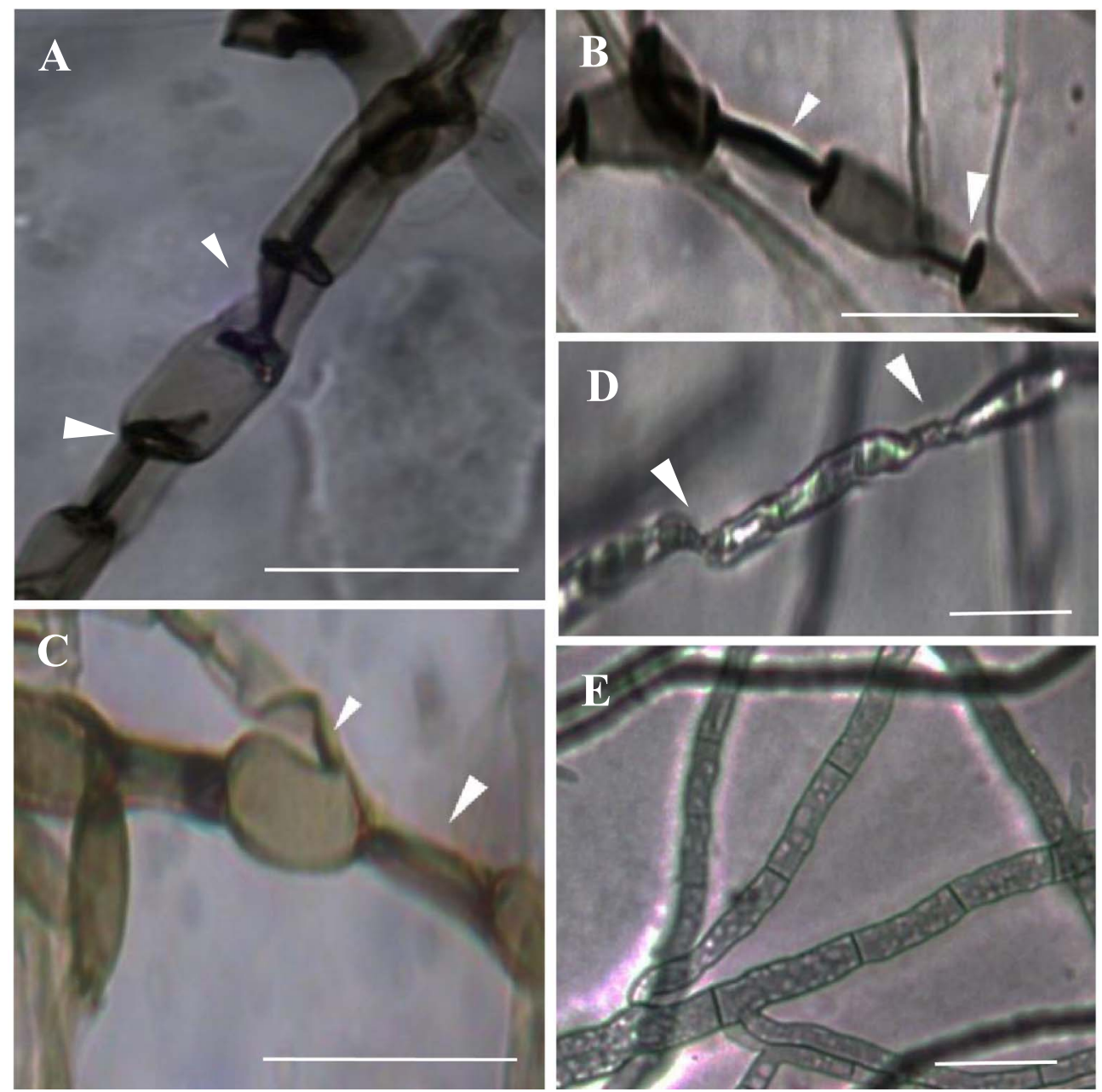

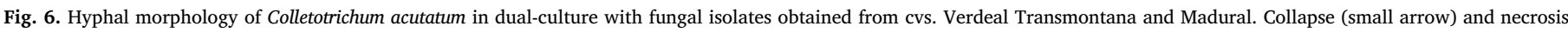

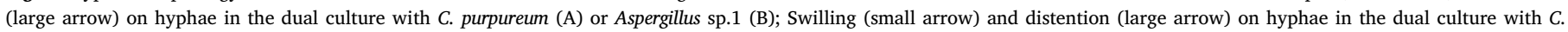
globosum (C); Collapse and twisting of hyphae in the dual culture with A. westerdijkiae (D); Normal hyphae (E) in control plates. Bar = $15 \mu \mathrm{m}$.

was evaluated for the first time. Most fungal OTUs identified in both cultivars belong to genera comprising relevant pathogens of other plant species (e.g. Alternaria, Gibberella, Lewia, Biscogniauxia and Cytospora), but also of olive tree. For instances, Neofabraea vagabunda, the causal agent of leprosy of olive (Romero et al., 2016), was detected in high abundance in olives from cv. Verdeal Transmontana as endophyte. The abundant detection of $N$. vagabunda on asymptomatic fruits was quite surprisingly, suggesting a conspicuous colonization of olive tissues by this fungus before the appearance of symptoms. At lower abundance, other olive fungal pathogens represented by Colletotrichum spp., a genus that contains different species associated with olive anthracnose (Schena et al., 2014), were also detected as endophyte in olives from cv. Madural. In this work was additionally found a number of nonpathogenic OTUs, being some of them described in the literature as presenting the useful features of antagonism against phytopathogens, such as Chaetomium globosum (Awad et al., 2014), Epicoccum nigrum (Fávaro et al., 2012), Penicillium brevicompactum (Jackson et al., 1997) and Trichoderma koningii (Reddy et al., 2014), and/or entomopathogenecity (e.g. Aspergillus westerdijkiae; Baggio et al., 2016). There is very limited information on isolation and characterization of epiphytes and endophytes from fresh olives. As far as we known, only Abdelfattah et al. (2015) have recently evaluated the diversity of fungi associated to olive fruits of the Italian cultivar "Ottobratica" through metabarcoding. Although the difficult in comparing our results with this study, in which fungal diversity was assessed using culture-independent technique, both studies found that olive fruits were numerically dominated by
Ascomycota and comprised a number of OTUs belonging to the genera Cladosporium, Alternaria and Colletotrichum.

Overall, the total species number and fungal isolates was greater on surface than in the interior of olive of both cultivars (up to 1.8- and 9.1fold higher, respectively). Very few studies have compared endophytic and epiphytic fungi, especially in fresh fruits. Comparison of epiphytic and endophytic fungal communities on single leaves of deciduous shrubs (Osono, 2007) or of woody plants (Osono, 2008), supporting our findings. The higher number of epiphytes recovered suggests that the external environment suits the fungal population colonizing the surface of olive fruit, despite the harsh environmental conditions that typify this habitat (e.g. harmful UV light, high temperatures, low humidity, osmotic stress, and scarcity of nutrients) (Rastogi et al., 2013); whereas internal microenvironment of olive fruits appears to restricts the fungal population to a certain extent, probably due to the presence of antimicrobials in their tissues (e.g. phenolic compounds) and/or host plant defense reactions (Rastogi et al., 2013). Similarly, the composition of fungal communities inhabiting the surface of olives was completed different from that found in internal olive tissues, a pattern which has also been noted in previous studies performed in leaves (Osono, 2007, 2008). Only Cladosporium cladosporioides, out of the 27 fungal OTUs recovered in this study, was common to both fungal floras. The most abundant endophytes were the phytopathogens Gibberella baccata and Neofabraea vagabunda. The former specie, which is associated with dieback, cankers and bud rots of woody trees and shrubs (Chen et al., 2016), has also been commonly encountered as 


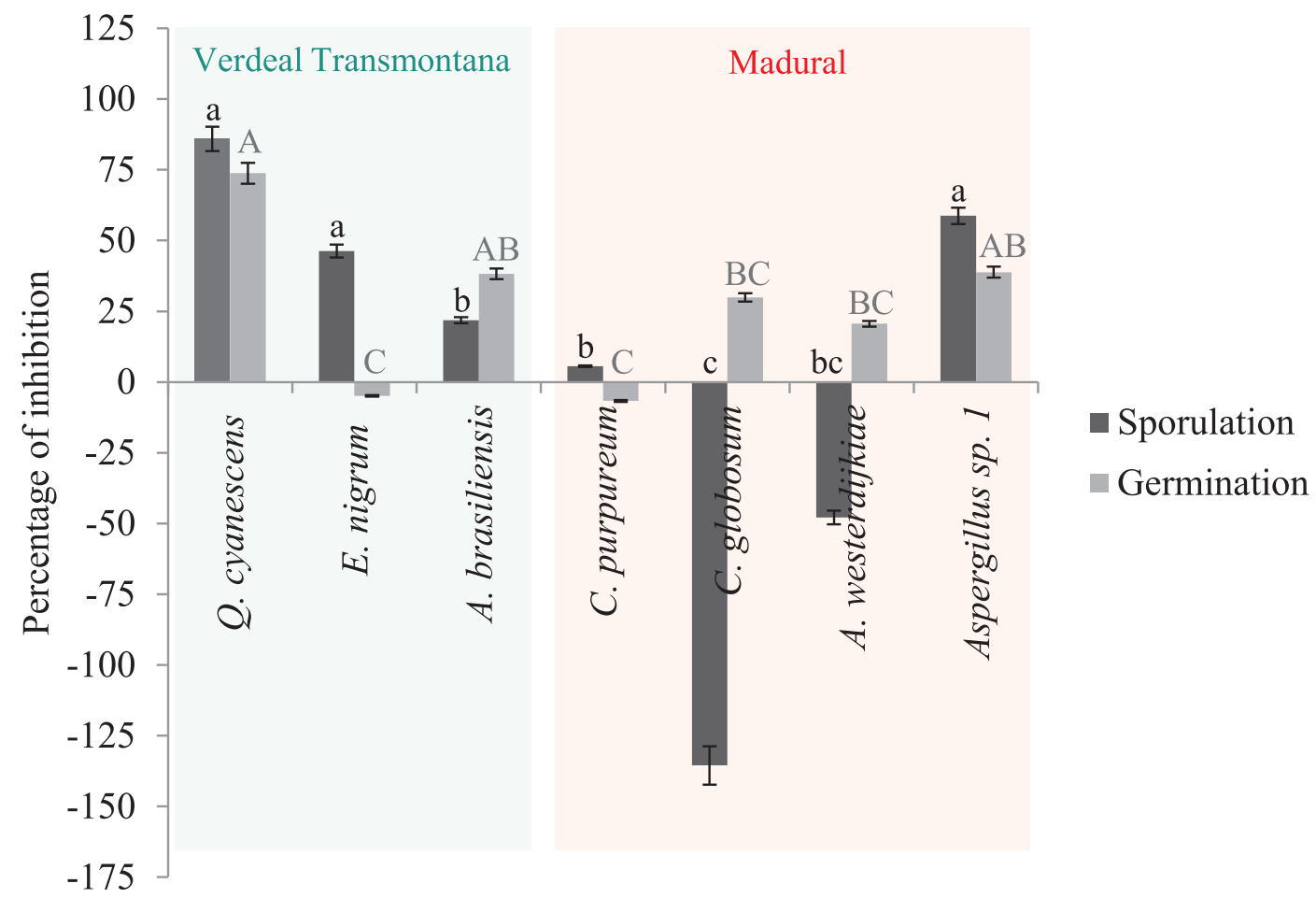

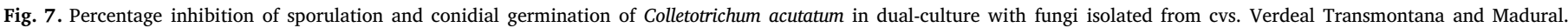

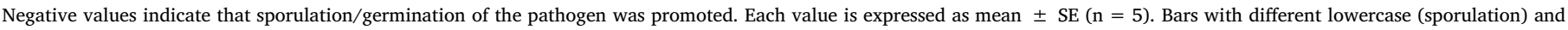
uppercase (germination) letters indicate significant differences $(\mathrm{p}<0.05)$.

endophytes of other woody plants, such as Taxus spp. (Xiong et al., 2013). The most abundant epiphytes were C. cucumerinum and $B$. mediterranea, both plant-pathogens that cause scab of cucurbits on cucumbers (Kwon et al., 1999) and charcoal canker in particular on the genus Quercus (Moricca et al., 2016), respectively.

The diversity of epiphytic fungi in olives surface was found to be different in the two cultivars, but their communities' composition was not. In the three olive orchards surveyed, trees of the two cultivars are relatively close to each other, and thus exposed to the same aerial inoculum, which could account for the high similarity found in the epiphytic fungal communities between cultivars. Despite this high similarity, we have noticed that several epiphytes preferred either olives from cv. Madural (Chaetomium spp.) or from cv. Verdeal Transmontana (Cytospora spp., Epicoccum spp. and Quambalaria spp.). Thus, being exposed to the same fungal inoculum, olive tree cultivar seems also to shape the detected epiphytic fungal communities. A similar finding was reported for fungi colonizing the leaf surfaces of different cultivars of the same woody plant species (Kembel and Mueller, 2014) or berries of several grapevine varieties (Bokulich et al., 2013). In contrast, the composition of endophytic fungal communities harbored by olives was found to be different between cultivars, as reported in other studies (Gazis and Chaverri, 2010). The exclusive presence of $N$. vagabunda with high relative abundance in $\mathrm{cv}$. Verdeal Transmontana was the major contributor for the differences found in the endophytic fungal communities among cultivars. As mentioned earlier, the cultivars Verdeal Transmontana and Madural, have different susceptibilities towards diseases (in particular to olive anthracnose), which might be an important factor in shaping not only phytopathogen (e.g. N. vagabunda and Colletotrichum spp., were virtually exclusively present in cv. Verdeal Transmontana and Madural, respectively), but also entire fungal communities. However, no clear correlation was found between known disease resistances in two cultivars and the endophytic fungal communities (data not shown). Similarly, Pan et al. (2008) found that resistance towards Uromyces maydis in genetically distinct maize recombinant inbred lines did not correlate with endophyte communities.
Dual-culture experiments showed that all the seven fungal tested were able to reduced $C$. acutatum growth, being the most potent inhibitors the epiphytes $C$. globosum ( $\mathrm{CI}=71.3)$, A. westerdijkiae $(\mathrm{CI}=57.9)$ and $E$. nigrum $(\mathrm{CI}=57.2)$. Previous studies have also reported the potential of $C$. globosum and E. nigrum to reduce the growth of several phytopathogenic fungi (e.g. Aggarwal, 2015; Biswas et al., 2012; Fávaro et al., 2012; Lahlali and Hijri, 2010); however, this is the first work showing an effective antagonist activity of these two species against $C$. acutatum. Similarly, studies examining antagonistic effect of $A$. westerdijkiae against phytopathogens have never been performed, being only tested its entomopathogenic properties against Periplaneta americana (Baggio et al., 2016). The suppression of $C$. acutatum growth by fungal isolates tested was mediated mostly at distance and/or in its vicinity, and in less extended following contact. Although initially all fungi formed an inhibition zone, they continued to grow at a higher rate than the $C$. acutatum leading a direct contact between colonies. Therefore, the investigated fungi might probably suppress C. acutatum growth either through general inhibition (e.g. involvement of inhibitory substances) and competition for space (due to their advantage of rapid growth).

Most of the fungal isolates tested strongly reduced $C$. acutatum germination, and all of them induced morphological abnormalities in $C$. acutatum hyphae, highlighting the antagonist effectiveness of the isolates investigated. The observed effects were presumably the result of bioactive compounds (such as lytic enzymes or inhibitory compounds), secreted by the antagonistic isolates, that may act on $C$. acutatum. In fact, both $C$. globosum and $E$. nigrum have been known to produce many bioactive compounds (such as chaetoglobosins, chaetomin and chaetoviridins, and flavipin, respectively) that have revealed the capacity to inhibit colony growth or germination of several phytopathogenic fungi (Aggarwal, 2015; Biswas et al., 2012; Madrigal and Melgarejo, 1995). Both species are also known to produce lytic enzymes (e.g. xylanase and $\beta$ 1,3-glucanase) (Aggarwal, 2015; Fávaro et al., 2012).

During interaction the mycelium of $C$. acutatum become yellow/ brown pigmented in the contact zone with E. nigrum, A. brasiliensis, $C$. 
purpureum, C. globosum and Aspergillus sp.1. Mycelium pigmentation may indicate the formation of melanin and melanin-like compounds. Many fungi produce melanins to protect cells against environmental factors such as visible and ultraviolet light, lytic enzymes, toxic metals and antagonistic microorganisms (Butler et al., 2009). Therefore the formation of pigments in C. acutatum mycelium may be a mechanism of the pathogen to protect hyphae from the antagonistic fungi by preventing access by cell wall degrading enzymes, a finding also reported by others (Larran et al., 2016). The lack of significant correlation between the sources of investigated fungal isolates (i.e. olive tree cultivars) and antagonist effectiveness (data not shown), suggested that the antagonistic activity of the isolates exhibited under in vitro conditions depended much more on the fungi than on their provenances.

\section{Conclusions}

Host plant genotype at cultivar level seems to play a significant role in the selection of fungal communities associated to their fruits, particularly in inner tissues of olives. This selection might have important implications for olive tree health and growth since phyllosphere microorganisms are known to have a variety of positive or negative effects (Heijden and Hartmann, 2016). Olives of both cultivars harbor distinct fungal communities containing pathogenic (e.g. Colletotrichum spp., $N$. vagabunda), and potentially antagonists. In fact, all the seven fungal tested exhibited antagonistic activity against $C$. acutatum. Further investigations are required to better ascertain the role of these fungi under natural conditions, and to assess their potential in the biocontrol of $C$. acutatum. The studies detailed here indicate that $Q$. cyanescens, E. nigrum and C. globosum holds great promise for this purpose.

\section{Acknowledgments}

This work was funded by National Funds through FCT - Foundation for Science and Technology under the project "EndoBio - Isolation and selection of endophytic fungi from olive tree for the biological control of Colletotrichum acutatum and Verticillium dahliae" (PTDC/AGR-PRO/ 4354/2012).

\section{Appendix A. Supplementary data}

Supplementary data associated with this article can be found, in the online version, at http://dx.doi.org/10.1016/j.biocontrol.2017.03.011.

\section{References}

Abdelfattah, A., Nicosia, M.G.L.D., Cacciola, S.O., Droby, S., Schena, L., 2015. Metabarcoding analysis of fungal diversity in the phyllosphere and carposphere of olive (Olea europaea). PLoS ONE 10, e0131069.

Aggarwal, R., 2015. Chaetomium globosum: a potential biocontrol agent and its mechanism of action. Indian Phytopath. 68, 8-24.

Agler, T.A., Ruhe, J., Kroll, S., Morhenn, C., Kim, S.T., Weigel, D., 2016. Microbial hub taxa link host and abiotic factors to plant microbiome variation. PLoS Biol. 14, 1-31.

Awad, N.E., Kassem, H.A., Hamed, M.A., El-Naggar, M.A.A., El-Feky, A.M.M., 2014. Bioassays guided isolation of compounds from Chaetomium globosum. J. Med. Mycol. 24, e35-e42.

Baggio, M.V., Ferreira, M.C., Monteiro, A.C., Junior, W.M., Lemos, M.V.F., 2016. Pathogenicity of Aspergillus westerdijkiae to females and oothecae of Periplaneta Americana. Ciência Rural 46, 20-25.

Biswas, S.K., Aggarwal, R., Srivastava, K.D., Gupta, S., Dureja, P., 2012. Characterization of antifungal metabolites of Chaetomium globosum Kunze and their antagonism against fungal plant pathogens. J. Biol. Control. 26, 70-74.

Boddy, L., 2000. Interspecific combative interactions between wood-decaying basidiomycetes. FEMS Microbiol. Ecol. 31, 185-194.

Bokulich, N., Thorngate, J.H., Richardson, P.M., Mills, D.A., 2013. Microbiol biogeography of wine grapes is conditioned by cultivar, vintage, and climate. Proc. Natl. Acad. Sci. 111, e139-e148.

Bray, J.R., Curtis, J.T., 1957. An ordination of the upland forest communities of Southern Wisconsin. Ecological Monographs 27.
Butler, M.J., Gardiner, R.B., Day, A.W., 2009. Melanin synthesis by Sclerotinia sclerotiorum. Mycologia 101, 296-304.

Cacciola, S.O., Faedda, R., Sinatra, F., Agosteo, G.E., Schena, L., Frisullo, S., Magnano, di S.L.G., 2012. Olive Anthracnose. J. Plant Pathol. 94, 29-44.

Chen, C., Gerard, J.M.V., Sun, G., Groenewald, J.Z., Crous, P.W., 2016. Redefining common endophytes and plant pathogens in Neofabraea, Pezicula, and related genera. Integrative taxonomy - Uncovering fungal diversity. Fungal Biol. 120, 1291-1322.

Colwell, R.K., 2013. EstimateS: Statistical estimation of species richness and shared species from samples Version 9. User's Guide and application.

Cray, J.A., Houghton, J.D.R., Cooke, L.R., Hallsworth, J.E., 2015. A simple inhibition coefficient for quantifying potency of biocontrol agents against plant-pathogenic fungi. Biol. Control 81, 93-100.

Fávaro, L.C.L., Sebastianes, F.L.S., Araújo, W.L., 2012. Epicoccum nigrum P16, a sugarcane endophyte, produces antifungal compounds and induces root growth. PLOS ONE 7, e36826.

Gazis, R., Chaverri, P., 2010. Diversity of fungal endophytes in leaves and stems of wild rubber trees (Hevea brasiliensis) in Peru. Fungal Ecol. 3, 240-254.

Heijden, M.G.A., Hartmann, M., 2016. Networking in the plant microbiome. PLoS Biol. 14, e1002378.

Henderson, P.A., Seaby, R.M.H., 2014. Community Analysis Package Version 5. Pisces Conservation Ltd, Lymington, UK.

Hermoso, M., Uceda, M., Frias, L., Beltrán, G., 2001. Maduración. In: Barranco, D., Fernández-Escobar, R., Rallo, L. (Eds.), El Cultivo Del Olivo $4^{\mathrm{a}}$ edicion. Ediciones Mundi-Prensa, Madrid, pp. 153-170.

Jackson, A.J., Walters, D.R., Marshall, G., 1997. Antagonistic interactions between the foliar pathogen Botrytis fabae and isolates of Penicillium brevicompactum and Cladosporium cladosporioides on faba beans. Biol. Control 8, 97-106.

Kembel, S.W., Mueller, R.C., 2014. Plant traits and taxonomy drive host associations in tropical phyllosphere fungal communities. Botany 92, 303-311.

Kwon, M.-K., Hong, J.-R., Cho, B.-H., Ki, U.-K., Kim, K.-C., 1999. A Scab disease caused by Cladosporium cucumerinum on watermelon seedlings. Plant Pathol. J. 15, 72-75.

Lahlali, R., Hijri, M., 2010. Screening, identification and evaluation of potential biocontrol fungal endophytes against Rhizoctonia solani AG3 on potato plants. FEMS Microbiol. Lett. 311, 152-159.

Larran, S., Simón, M.R., Moreno, M.V., Siurana, M.P.S., Perelló, A., 2016. Endophytes from wheat as biocontrol agents against tan spot disease. Biol. Control 92, 17-23.

Lindow, S.L., Brandl, M.T., 2003. Microbiology of the phyllosphere. Appl. Environ. Microbiol. 69, 1875-1883.

Madrigal, C., Melgarejo, P., 1995. Morphological effects of Epiccocum nigrum and its antibiotic flavipin on Monilinia laxa. Can. J. Bot. 73, 425-431.

Magurran, A.E., 2004. Measuring Biological Diversity. Blackwell Publishing, Oxford, UK.

Moral, J., Xaviér, C., Roca, L.F., Romero, J., Moreda, W., Trapero, A., 2014. La Antracnosis del olivo y su efecto en la calidad del aceite. Grasas Aceites 65, e028.

Moricca, S., Linaldeddu, B.T., Ginetti, B., Scanu, B., Franceschini, A., Ragazzi, A., 2016. Endemic and Emerging Pathogens Threatening Cork Oak Trees: Management Options for Conserving a Unique Forest Ecosystem. Plant Dis. 100, 2184-2193.

Oliveira, I., Pereira, J.A., Lino-Neto, T., Bento, A., Baptista, P., 2012. Fungal diversity associated to the olive moth, Prays oleae Bernard: a survey for potential entomopathogenic fungi. Microb. Ecol. 63, 964-974.

Osono, T., 2008. Endophytric and epiphytic phyllosphere fungi of Camellia japonica: seasonal and leaf age-dependent variations. Mycologia 100, 387-391.

Osono, T., 2007. Endophytic and epiphytic phyllosphere fungi of red-osier dogwood (Cornus stolonifera) in British Columbia. Mycoscience 48, 47-52.

Pan, J.J., Baumgarten, A.M., May, G., 2008. Effects of host plant environment and Ustilago maydis infection on the fungal endophyte community of maize (Zea mays). New Phytol. 178, 147-156.

Rastogi, G., Coaker, G.L., Leveau, J.H.J., 2013. New insights into the structure and function of phyllosphere microbiota through high-throughput molecular approaches. FEMS Microbiol. Lett. 348, 1-10.

Reddy, B.N., Saritha, K.V., Hindumathi, A., 2014. In vitro screening for antagonistic potential of seven species of Trichoderma against different plant pathogenic fungi. Res. J. Biol. 2, 29-36.

Romero, J., Raya, M.C., Roca, L.F., Moral, J., Trapero, A., 2016. First report of Neofabraea vagabunda causing branch cankers on olives in Spain. Plant Dis. 100, 527.

Schena, L., Mosca, S., Cacciola, S.O., Faedda, R., Sanzani, S.M., Agosteo, G.E., Sergeeva, V., Magnano, di S.L.G., 2014. Species of the Colletotrichum gloeosporioides and C. boninense complexes associated with olive anthracnose. Plant. Pathol. 63, 437-446.

Seaby, R.M., Henderson, P.A., 2006. Species Diversity and Richness Version 4. Pisces Conservation Ltd., Lymington, England.

Soares, M.E., Pereira, J.A., Bastos, M.L., 2006. Validation of a method to quantify copper and other metals in olive fruits by ETAAS. Application to the residual metal control after olive tree treatments with different copper formulations. J. Agric. Food Chem. 54, 3923-3928.

Talhinhas, P., Mota-Capitão, C., Martins, S., Ramos, A.P., Neves-Martins, J., GuerraGuimarães, L., Várzea, V., Silva, M.C., Sreenivasaprasad, S., Oliveira, H., 2011. Epidemiology, histopathology and aetiology of olive anthracnose caused by Colletotrichum acutatum and C. gloeosporioides in Portugal. Plant. Pathol. 60, 483-495.

Vorholt, J.A., 2012. Microbial life in the phyllosphere. Nat. Rev. Microbiol. 10, 828-840.

Weisstein, E.W., 2013. "Prolate Spheroid." From MathWorld - A Wolfram Web Resource. Available at: http://mathworld.wolfram.com/ProlateSpheroid.html [accessed November 30, 2015].

Xiong, Z.Q., Yang, Y.Y., Zhao, N., Wang, Y., 2013. Diversity of endophytic fungi and screening of fungal paclitaxel producer from Anglojap yew. Taxus x media. BMC Microbiol. 13, 1-10. 